\title{
How secondhand smoke exposure affects tobacco use and smoking susceptibility of adolescents: Sex and school differences
}

\author{
Xiaochen Yang ${ }^{1 *}$, Zhongheng Yan ${ }^{2 *}$, Gang X $u^{1}$, Yinliang Tan' ${ }^{1}$ Jingfen Zhu ${ }^{1}$
}

\begin{abstract}
INTRODUCTION Secondhand smoke (SHS) exposure affects tobacco related health behaviors during adolescence and persists into adulthood. This study aimed to investigate the influence of SHS exposure on tobacco use among adolescents stratified by school and gender, and provide recommendations for controlling tobacco use in youth.

METHODS Through stratified random cluster sampling, 12278 selected students (aged 13-18 years) from schools in China were administered questionnaires. Multiple logistic regression was used to analyze whether SHS exposure would increase the smoking risk and susceptibility of adolescents.

RESULTS The prevalence of SHS exposure among the participating students was $74.8 \%$. Adolescents exposed to SHS were at higher odds of being susceptible and currently smoking. Students with SHS exposure at both home and public places accounted for $36.6 \%$, greatly increasing the current smoking risk and smoking susceptibility. Home SHS exposure had greater impact on the current tobacco use of boys ( $\mathrm{OR}=2.13 ; 95 \% \mathrm{CI}: 1.50-3.03)$ and junior school students $(\mathrm{OR}=4.67$; 95\% CI: 2.41-9.06). Exposure from public places increased the risk of current smoking in boys $(\mathrm{OR}=4.20 ; 95 \%$ CI: 2.31-7.65) and smoking susceptibility of vocational school students $(\mathrm{OR}=1.51 ; 95 \% \mathrm{CI}: 1.07-2.15)$. Students with highlevel exposure to SHS had 2.25 times higher odds of e-cigarette use.

CONCLUSIONS The prevalence of SHS exposure is still high among adolescents in China and is associated with increased risk for tobacco use regardless of gender and school level. Effective smoke-free strategies should be developed and strictly implemented. Boys and junior school students constitute vulnerable populations exposed to SHS at home.
\end{abstract}

AFFILIATION
1 School of Public Health,
Shanghai Jiao Tong University
School of Medicine, Shanghai,
People's Republic of China
2 The Ninth People's Hospital,
Shanghai Jiao Tong University
School of Medicine, Shanghai,
People's Republic of China
${ }^{*}$ Contributed equally
CORRESPONDENCE TO
Jingfen Zhu. School of Public
Health, Shanghai Jiao Tong
University School of Medicine,
227 South Chongqing Road,
Shanghai 200025, People's
Republic of China.
E-mail: zhujingfenjt@shsmu.
edu.cn

KEYWORDS

secondhand smoke, tobacco use, smoking susceptibility, adolescent, gender

Received: 16 May 2021

Revised: 1 July 2021

Accepted: 8 July 2021

\section{INTRODUCTION}

Tobacco use is currently one of the most serious public health threats worldwide. Tobacco use and related behaviors, including secondhand smoke (SHS) exposure, smoking susceptibility, and exposure to tobacco advertising, harm health and impose a heavy economic burden on society ${ }^{1-3}$. Smoking susceptibility precedes smoking behavior and is defined as not taking a firm decision against cigarette smoking ${ }^{4}$. It acts as a predictor of smoking experimentation and smoking status, with adolescents less likely to respond to tobacco prevention programs ${ }^{5}$. Prior research has indicated that smoking susceptibility is used as a significant independent predictor of future initiation into tobacco use ${ }^{6}$.

Despite the approval of the World Health Organization (WHO) Framework Convention on Tobacco Control (FCTC) in 2005, China is now the world's leading nation in both tobacco production and consumption $^{7}$, with more than 300 million smokers 
and 740 million non-smokers exposed to $\mathrm{SHS}^{8}$. Over the past few decades, China has been carrying out tobacco prevention interventions including cigarette sale age restrictions, media campaigns, and school health education courses that target middle school and high school students ${ }^{9}$. The current smoking prevalence among adolescents in junior high schools was $3.9 \%$ in 2019 and the rates for senior and vocational high school students were $8.6 \%$ and $14.7 \%$, respectively ${ }^{10}$. The prevalence of smoking among junior high school students in 2019 was lower than that in $2014(6.9 \%)^{10}$, which might prove tobacco control efforts effective, but this rate was still high.

The smoking behavior of adolescents is affected by personal, psychological, and social factors such as gender, smoking behavior of peers or family members and social environment ${ }^{11,12}$. SHS exposure is one of the important risk factors that might increase susceptibility to smoking and nicotine dependence among those who have begun smoking ${ }^{13}$. Prior studies have indicated that SHS could influence tobacco use and smoking behaviors ${ }^{14-16}$. Review studies found that SHS exposure was associated with increased susceptibility and initiation of smoking ${ }^{17}$. According to one of the nicotine dependence theories, SHS exposure could result in repeated nicotine exposure and tolerance to nicotine's adverse effects, thereby contributing to nicotine-induced behavioral changes ${ }^{18}$.

Research has shown that an adolescent who smokes a first cigarette is more likely to continue into adulthood and have greater difficulty quitting ${ }^{19}$. Considering the harmful effects of smoking in adolescents, how SHS exposure induces adolescents to initiate smoking warrants further clarification. In a high-density urban setting, the passive exposure to SHS in adolescents can be much higher than the smoking prevalence of the general population ${ }^{20}$. According to a study conducted in the United States, homes are the settings where the highest percentage of non-smokers have reported daily SHS exposure ${ }^{12}$. Compared with adults, private settings are probably major and neglected sources of exposure for children and adolescents. Therefore, identifying the characteristics of SHS exposure among adolescents is of great importance. The conflicting reports on the disparities of gender and school in adolescent smoking behavior support the need for more research with regard to the relationship between SHS exposure and tobacco use $\mathrm{e}^{11,21,22}$. How specific levels of sources of exposure affect young people of different genders also needs more research, since more boys than girls become more susceptible to initiating smoking as they age $^{23}$.

Less is known about how the levels or sources of SHS exposure affect e-cigarette use, which currently presents a high prevalence among adolescents. Prior studies have proven that SHS exposure at home mediates family smoking and e-cigarette use of adolescents ${ }^{24}$. The correlations between SHS exposure and e-cigarette use might present diversified stratification by gender and school.

A number of studies have examined the factors associated with tobacco use and initiation of smoking among adolescents. But the specific relationship between SHS exposure and tobacco related behaviors among those aged 13-18 years is scant and should be examined. With the high prevalence of SHS exposure and harmful role it plays in tobacco related behaviors in adolescents, the current study aimed to investigate the relationship between SHS exposure and tobacco use behaviors stratified by sex and school among a representative sample of adolescents in Shanghai, China. This study should assist in identifying adolescents who are at high risk of smoking initiation and inform interventions to improve targeted enforcement of smoke-free policies.

\section{METHODS}

\section{Study population and data collection}

This study was a cross-sectional study with two-stage stratified cluster sampling to select representative samples of school students aged 13-18 years in Shanghai from September 2017 to January 2018. In the first stage, the sixteen selected districts were stratified according to urban and rural areas, and four districts were randomly selected. Huangpu and Putuo were selected as central urban areas, and Minhang and Jiading as non-central urban areas. In the second stage, all schools in these four districts were further stratified based on school type and 33 schools were randomly selected. A total of 12422 students from 33 schools in 4 districts were invited to participate in this study, and 12278 (98.8\%) valid questionnaires were included in the analysis.

The self-administered questionnaire was adapted from the WHO Global Youth Tobacco Survey. The 
online questionnaires were administered during regular class sessions and students were asked to complete them anonymously and independently. Trained research members briefed students on the details of the items in the questionnaire. The other details regarding the panel have been published ${ }^{25}$. This study was approved by the Ethics Committee of the Shanghai Jiao Tong University (SJUPN-201703; approved on December 5, 2017).

\section{Measures}

We defined participants as current smokers if they reported smoking in the last 30 days. Ever smokers were defined as those who had ever tried a cigarette but not in the past 30 days. The susceptibility to smoking in the future among never smokers was assessed by two questions: 'Do you think you will smoke a cigarette in the next year?' and 'If your best friend offers you a cigarette, may you smoke it?'. Participants who answered 'definitely not' to both questions were classified as non-susceptible to smoking while the others were classified as susceptible. Use of electronic cigarettes were measured using the question: 'Have you tried e-cigarettes (even one puff)?'; with those who responded 'yes' defined as ever users.

Exposure to SHS at home, in indoor public places, and at outdoor areas was measured by five-point ordinal scale: '0 days', ' $1-2$ days', '3-4 days', '5-6 days' and 'all 7 days' during the past seven days. Those who reported ' 0 days' to all three places were identified as not having SHS exposure. Respondents who were exposed to SHS in public places were referred to those reporting more than 0 days exposure to indoor or outdoor places. To investigate the effects of different exposure levels, the total score of these three questions was used as a continuous variable and divided equally into low, medium, and high SHS exposure levels, respectively. To create distinct groups based on the source of SHS exposure, adolescents were categorized into the following four groups: 1 ) no SHS exposure, 2) only exposure at home, 3) only exposure in public places, and 4) SHS exposure in both places.

Sociodemographic covariates included age, gender, school type, residence status, household registration (local, non-local), pocket money, academic performance and smoking status of close friends and parents.

\section{Statistical analysis}

All statistical analyses were performed using IBM SPSS 22.0 and the complex sample analysis was used. A weighting factor was applied to each student record to adjust for non-response and for varying probabilities of selection including selection probability of districts, the number of schools in each district, and the number of students in each school. SHS exposure and tobacco use conditions were presented as percentages. Chisquared analysis was used to test the relationship between gender and school type both at home as well as in public places. Multiple logistic regression was used to explore the association between SHS exposure and current smoking status, smoking susceptibility as well as e-cigarette use among adolescents. Sex, age, school type, district, boarding, local, GPA, pocket money, friends' smoking and parents' smoking were included as covariates. The trend test was used to analyze the relationship between SHS exposure level and tobacco use behaviors. Odds ratios (ORs) and 95\% confidence intervals (CIs) are reported. Differences were considered to be statistically significant for $\mathrm{p}<0.05$.

\section{RESULTS}

Among the respondents, $51.6 \%$ were males and $48.4 \%$ were females. The proportion of junior high school students, senior high school students and vocational school students was $61.99 \%, 23.67 \%$ and $14.34 \%$, respectively, and this was similar to the composition of students in Shanghai. Current smokers and e-cigarette users were $2.52 \%$ and $7.67 \%$ of the investigated students while smoking susceptibility among nonsmokers was $7.67 \%$ (Table 1 ).

The prevalence of reported SHS exposure among adolescents in our sample was $74.8 \%$, and that at home and public places was $41.5 \%$ and $69.9 \%$, respectively. The rate of SHS exposure in indoor public places was $59.3 \%$ and at outdoor public places was $63.5 \%$. More male students reported being exposed to high levels of SHS $(30.4 \%)$ compared to females $(26.9 \%, \mathrm{p}<0.001)$. Vocational students with SHS exposure both at home and public places accounted for $43.4 \%$, which was significantly higher than junior and senior high school students $(34.7 \%$ and $37.4 \%, \mathrm{p}<0.001)$. The prevalence of overall SHS exposure among adolescents from different schools showed significant differences $\left(X^{2}=7.97, p<0.05\right)$, and similarly that of home $\left(X^{2}=\right.$ 
Table 1. Basic characteristics of survey subjects ( $N=12278)$

\begin{tabular}{|c|c|c|c|}
\hline & \multicolumn{2}{|c|}{ Weighted } & \multirow{2}{*}{$\begin{array}{c}\text { Unweighted } \\
\text { Mean (95\% CI) }\end{array}$} \\
\hline & Mean $(95 \%$ CI) & Number & \\
\hline \multirow[t]{2}{*}{ Age (years) } & $14.28(14.23-14.31)$ & 670050 & $14.62(14.58-14.66)$ \\
\hline & $\%(95 \%$ CI $)$ & Number & $n(\%)$ \\
\hline \multicolumn{4}{|l|}{ Type of school } \\
\hline Junior high school & $61.99(61.13-62.85)$ & 415377 & $6462(52.63)$ \\
\hline Senior high school & $23.67(22.88-24.48)$ & 158593 & $2475(20.16)$ \\
\hline Vocational high school & $14.34(13.86-14.83)$ & 96080 & $3341(27.21)$ \\
\hline \multicolumn{4}{|l|}{ District } \\
\hline Urban & $33.36(33.13-33.60)$ & 223534 & 4042 (32.92) \\
\hline Suburban & $66.64(66.40-66.87)$ & 446516 & $8236(67.08)$ \\
\hline \multicolumn{4}{|l|}{ Gender } \\
\hline Male & $51.6(50.69-52.52)$ & 345778 & $6419(52.28)$ \\
\hline Female & $48.4(47.48-49.31)$ & 324272 & 5859 (47.72) \\
\hline \multicolumn{4}{|l|}{ Residence } \\
\hline Local & 72.21 (71.39-73.02) & 483864 & 8755 (71.31) \\
\hline Non-local & $27.79(26.98-28.61)$ & 186186 & 3523 (28.69) \\
\hline \multicolumn{4}{|l|}{ Boarding in school } \\
\hline Yes & $13.55(13.00-14.12)$ & 90791 & $2302(18.75)$ \\
\hline No & $86.45(85.88-87.00)$ & 579260 & 9976 (81.25) \\
\hline \multicolumn{4}{|c|}{ Monthly allowance (RMB) } \\
\hline$<200$ & $61.23(60.35-62.11)$ & 173882 & 7011 (57.10) \\
\hline $200-600$ & $25.95(25.17-26.75)$ & 85865 & 3401 (27.70) \\
\hline$>600$ & $12.81(12.24-13.41)$ & 670050 & $1866(15.20)$ \\
\hline \multicolumn{4}{|l|}{ GPA } \\
\hline Top 25\% & $32.96(32.11-33.83)$ & 220860 & 3924 (31.96) \\
\hline Average & $46.28(45.38-47.20)$ & 310129 & 5712 (46.52) \\
\hline Bottom 25\% & 20.75 (20.03-21.5) & 139061 & $2642(21.52)$ \\
\hline \multicolumn{4}{|l|}{ Parents' smoking } \\
\hline None & $36.56(35.68-37.44)$ & 244962 & 4377 (35.65) \\
\hline One & $59.25(58.35-60.14)$ & 396985 & 7364 (59.98) \\
\hline Both & $4.19(3.85-4.57)$ & 28103 & $537(4.37)$ \\
\hline \multicolumn{4}{|l|}{ Friends' smoking } \\
\hline None & $82.79(82.13-83.43)$ & 554740 & 9693 (78.95) \\
\hline Some & $15.16(14.55-15.79)$ & 101550 & 2274 (18.52) \\
\hline Most or all & $2.05(1.83-2.31)$ & 13760 & $311(2.53)$ \\
\hline \multicolumn{4}{|l|}{ Smoking status } \\
\hline Never & 92.12 (91.64-92.57) & 617244 & 11091 (90.33) \\
\hline Ever & $5.36(4.98-5.76)$ & 35893 & $780(6.35)$ \\
\hline Current & $2.52(2.28-2.80)$ & 16913 & $407(3.31)$ \\
\hline \multicolumn{4}{|l|}{ Smoking susceptibility } \\
\hline No & 92.33 (91.86-92.78) & 618651 & 11140 (90.73) \\
\hline Yes & 7.67 (7.22-8.14) & 51399 & $1138(9.27)$ \\
\hline \multicolumn{4}{|l|}{ E-cigarette use } \\
\hline No & 92.33 (91.86-92.78) & 618651 & 11524 (93.86) \\
\hline Yes & 7.67 (7.22-8.14) & 51399 & $754(6.14)$ \\
\hline
\end{tabular}

Cl: confidence interval. GPA: grade point average. RMB: 100 Chinese Renminbi about US\$15. 


\section{Research Paper}

Table 2. Secondhand smoke exposure rate among adolescents stratified by sex and school ( $\mathrm{N}=12278)$

\begin{tabular}{|c|c|c|c|c|c|c|c|c|c|c|}
\hline & \multicolumn{4}{|c|}{ Sex } & \multicolumn{5}{|c|}{ High school } & \multirow[t]{2}{*}{ Total } \\
\hline & Male & Female & \multirow[t]{2}{*}{$x^{2}$} & \multirow[t]{2}{*}{$p$} & Junior & Senior & Vocational & \multirow[t]{2}{*}{$x^{2}$} & \multirow[t]{2}{*}{$p$} & \\
\hline & $\%(95 \% \mathrm{CI})$ & $\%(95 \%$ CI $)$ & & & $\%(95 \%$ CI $)$ & $\%(95 \%$ CI $)$ & $\%(95 \% \mathrm{CI})$ & & & $\%(95 \% \mathrm{CI})$ \\
\hline No & $26.0(24.9-27.1)$ & $24.4(23.3-25.6)$ & 4.08 & 0.050 & $24.9(23.9-26.0)$ & $24.4(22.7-26.1)$ & $27.9(26.4-29.4)$ & 7.97 & 0.014 & $25.2(24.4-26.0)$ \\
\hline Yes & $74.0(72.9-75.1)$ & $75.6(74.4-76.7)$ & & & $75.1(74.0-76.1)$ & $75.6(73.9-77.3)$ & $72.1(70.6-73.6)$ & & & $74.8(74.0-75.6)$ \\
\hline \multicolumn{11}{|l|}{ SHS from home } \\
\hline Yes & $42.3(41.1-43.6)$ & $40.7(39.4-42.0)$ & & & $39.5(38.3-40.6)$ & $42.1(40.2-44.0)$ & $49.4(47.7-51.1)$ & & & $41.5(40.6-42.4)$ \\
\hline \multicolumn{11}{|c|}{ SHS from public places } \\
\hline No & $31.0(29.9-32.2)$ & $29.2(28.0-30.4)$ & 4.96 & 0.031 & $29.7(28.6-30.8)$ & $29.1(27.3-30.9)$ & $33.8(32.2-35.4)$ & 13.62 & 0.001 & $30.1(29.3-31.0)$ \\
\hline Yes & $69.0(67.8-70.1)$ & $70.8(69.6-72.0)$ & & & $70.3(69.2-71.4)$ & $70.9(69.1-72.7)$ & $66.2(64.6-67.8)$ & & & $69.9(69.0-70.7)$ \\
\hline \multicolumn{11}{|c|}{ SHS from indoor public places } \\
\hline No & $41.1(39.8-42.3)$ & $40.2(38.9-41.5)$ & 0.998 & 0.333 & 40.7 (39.5-41.9) & $40(38.1-41.9)$ & $41.6(40.0-43.3)$ & 1.177 & 0.501 & $40.7(39.8-41.6)$ \\
\hline Yes & $58.9(57.7-60.2)$ & $59.8(58.5-61.1)$ & & & $59.3(58.1-60.5)$ & $60(58.1-61.9)$ & $58.4(56.7-60.0)$ & & & $59.3(58.4-60.2)$ \\
\hline No & $37.6(36.4-38.8)$ & $35.4(34.1-36.6)$ & 6.626 & 0.013 & $37.1(36-38.3)$ & $33.5(31.7-35.4)$ & $38.7(37-40.3)$ & 15.945 & $<0.001$ & $36.5(35.6-37.4)$ \\
\hline Yes & $62.4(61.2-63.6)$ & $64.6(63.4-65.9)$ & & & $62.9(61.7-64)$ & $66.5(64.6-68.3)$ & $61.3(59.7-63)$ & & & $63.5(62.6-64.4)$ \\
\hline \multicolumn{11}{|l|}{ SHS level } \\
\hline Low & $36.4(35.2-37.6)$ & $36.2(34.9-37.4)$ & 25.89 & 0.000 & $36.9(35.7-38.1)$ & $34.7(32.9-36.6)$ & $36.4(34.8-38.0)$ & 12.37 & 0.009 & $36.3(35.4-37.2)$ \\
\hline Medium & $33.2(32.0-34.4)$ & $37.0(35.7-38.3)$ & & & $35.3(34.1-36.4)$ & $35.8(34.0-37.7)$ & $32.6(31.0-34.2)$ & & & $35.0(34.2-35.9)$ \\
\hline High & $30.4(29.2-31.6)$ & $26.9(25.7-28.0)$ & & & $27.8(26.8-28.9)$ & $29.5(27.7-31.3)$ & $31.0(29.5-32.6)$ & & & $28.7(27.9-29.5)$ \\
\hline \multicolumn{11}{|c|}{ SHS home and public } \\
\hline No & $26.0(24.9-27.1)$ & $24.4(23.3-25.6)$ & 14.80 & 0.003 & $24.9(23.9-26.0)$ & $24.4(22.7-26.1)$ & $27.9(26.4-29.4)$ & 111.78 & 0.000 & $25.2(24.4-26)$ \\
\hline Home only & $5.0(4.5-5.6)$ & $4.8(4.2-5.4)$ & & & $4.7(4.2-5.3)$ & $4.7(4-5.6)$ & $6.0(5.2-6.8)$ & & & $4.9(4.5-5.3)$ \\
\hline Public places only & $31.7(30.5-32.9)$ & $34.9(33.7-36.2)$ & & & $35.6(34.4-36.8)$ & $33.5(31.7-35.4)$ & $22.7(21.4-24.2)$ & & & $33.3(32.4-34.1)$ \\
\hline Both & $37.3(36.1-38.5)$ & $35.9(34.6-37.1)$ & & & 34.7 (33.6-35.9) & $37.4(35.5-39.3)$ & $43.4(41.8-45.1)$ & & & $36.6(35.7-37.5)$ \\
\hline
\end{tabular}


Table 3. ORs for current smoking by secondhand smoke exposure stratified by sex and school $(\mathrm{N}=12278)^{\mathrm{a}}$

\begin{tabular}{|c|c|c|c|c|c|c|c|}
\hline & \multirow{2}{*}{$\frac{\text { Now smoking }}{\%(95 \% \mathrm{CI})}$} & \multirow[t]{2}{*}{ Total } & \multicolumn{2}{|c|}{$\operatorname{sex}$} & \multicolumn{3}{|c|}{ High school } \\
\hline & & & Male & Female & Junior & Senior & Vocational \\
\hline \multicolumn{8}{|l|}{ SHS home } \\
\hline No (Ref.) & $1.2(1-1.4)$ & 1 & 1 & 1 & 1 & 1 & 1 \\
\hline Yes & $4.4(3.9-5.0)$ & $2.45(1.80-3.33)$ & $2.13(1.50-3.03)$ & $1.60(1.06-2.41)$ & 4.67 (2.41-9.06) & $1.34(0.71-2.53)$ & $2.56(1.74-3.76)$ \\
\hline \multicolumn{8}{|l|}{ SHS public } \\
\hline No (Ref.) & $1.2(0.9-1.6)$ & 1 & 1 & 1 & 1 & 1 & 1 \\
\hline Yes & $3.1(2.8-3.5)$ & $1.34(0.93-1.93)$ & $4.20(2.31-7.65)$ & $0.77(0.37-1.60)$ & $0.80(0.39-1.64)$ & $2.23(0.93-5.34)$ & $1.28(0.85-1.94)$ \\
\hline \multicolumn{8}{|l|}{ SHS level } \\
\hline Low (Ref.) & $1.1(0.8-1.4)$ & 1 & 1 & 1 & 1 & 1 & 1 \\
\hline Medium & $1.3(1.0-1.6)$ & $1.04(0.70-1.56)$ & $1.31(0.83-2.06)$ & $0.56(0.23-1.33)$ & $0.64(0.23-1.79)$ & 1.07 (0.38-2.99) & $1.30(0.84-2.01)$ \\
\hline High & $5.9(5.2-6.7)$ & $3.26(2.23-4.76)$ & $3.53(2.26-5.50)$ & $2.70(1.31-5.57)$ & 3.79 (1.64-8.76) & $4.01(1.56-10.3)$ & $2.65(1.73-4.04)$ \\
\hline$p$ for trend & & $<0.001$ & $<0.001$ & 0.013 & 0.001 & 0.003 & $<0.001$ \\
\hline SHS (cont.) & & $1.16(1.12-1.20)$ & 1.15 (1.10-1.19) & $1.19(1.11-1.27)$ & $1.23(1.13-1.33)$ & $1.21(1.12-1.30)$ & $1.09(1.05-1.13)$ \\
\hline \multicolumn{8}{|l|}{$\begin{array}{l}\text { SHS home } \\
\text { and public }\end{array}$} \\
\hline No (Ref.) & $1.3(1.0-1.7)$ & 1 & 1 & 1 & 1 & 1 & 1 \\
\hline Home only & $0.8(0.4-1.7)$ & $0.51(0.20-1.31)$ & $0.54(0.19-1.57)$ & $0.49(0.04-5.76)$ & $0.48(0.06-3.88)$ & N/A & $1.10(0.42-2.88)$ \\
\hline Public only & $1.1(0.8-1.4)$ & $0.82(0.52-1.30)$ & $1.03(0.61-1.74)$ & $0.40(0.16-1.03)$ & $0.28(0.09-0.86)$ & $1.52(0.54-4.24)$ & $0.91(0.54-1.55)$ \\
\hline Both & $4.9(4.3-5.5)$ & $2.63(1.71-4.05)$ & $2.77(1.68-4.57)$ & $2.59(1.15-5.82)$ & $2.51(1.02-6.17)$ & $2.50(0.89-7.05)$ & $2.85(1.78-4.58)$ \\
\hline
\end{tabular}

OR: odds ratio. a Model adjusted for age, sex, school type, district, boarding, local, GPA, pocket money, friends' smoking and parents' smoking.

$58.56, \mathrm{p}<0.001)$ and public places $\left(\chi^{2}=13.62, \mathrm{p}<0.01\right)$ (Table 2).

The associations between SHS exposure and the risk of current smoking and smoking susceptibility of adolescents are presented in Tables 3 and 4. Overall, the risk of adolescents' tobacco use behaviors was greater the higher the exposure level. Male students with high-level SHS exposure had higher current smoking risk (OR=3.53; 95\% CI: 2.26-5.50) and smoking susceptibility $(\mathrm{OR}=1.66$; 95\% CI: $1.18-$ 2.23 ) compared with female students. The current smoking risk was the highest in senior high school students with high-level SHS exposure $(\mathrm{OR}=4.01$; 95\% CI: 1.56-10.30) than other students. Vocational high school students were most susceptible to smoking ( $\mathrm{OR}=1.83 ; 95 \% \mathrm{CI}: 1.22-2.75)$. Analysis of continuous exposure to SHS showed that the risk of current smoking was increased for any gender and school with more SHS exposure. Exposure to SHS in only one place, whether home or public place, showed no significant relationship with tobacco use risk, while SHS exposure at both places greatly increased the students' current smoking risk and smoking susceptibility. Stratified by sex and school, home SHS exposure had a greater impact on the current smoking behavior and smoking susceptibility of boys $(\mathrm{OR}=2.13 ; 95 \% \mathrm{CI}: 1.50-3.03)$ and junior high school students (OR=4.67; 95\% CI: 2.41-9.06). SHS exposure in public places increased the current smoking risk in boys $(\mathrm{OR}=4.20$; 95\% CI: $2.31-7.65)$ and the smoking susceptibility of vocational school students (OR=1.51; 95\% CI: 1.07-2.15).

Additionally, the relationship between SHS exposure and e-cigarette use risk was analyzed (Table 5). The odds of e-cigarette use were 2.25 (95\% CI: 1.77-2.87) times higher in those who had high exposure to SHS. Female students with home SHS exposure $(\mathrm{OR}=1.82$; 95\% CI: $1.18-2.79)$ and high SHS exposure (OR=3.05; 95\% CI: 1.90-4.87) had higher e-cigarette use risk than males. Senior high school students with SHS exposure at public places (OR=2.32; 95\% CI: 1.26-4.29) and high SHS exposure $(\mathrm{OR}=4.32 ; 95 \%$ CI: $2.41-7.74)$ had higher e-cigarette use risk. Those reporting SHS exposure from both places had 1.89 (95\% CI: 1.44-2.47) times increased odds of using e-cigarettes. 


\section{Research Paper}

Table 4. ORs for future smoking intention by secondhand smoke exposure stratified by sex and school among never smokers (N=11091)

\begin{tabular}{|c|c|c|c|c|c|c|c|}
\hline & \multirow{2}{*}{$\begin{array}{c}\text { Smoking } \\
\text { susceptibility }\end{array}$} & \multirow[t]{2}{*}{ Total } & \multicolumn{2}{|c|}{ Sex } & \multicolumn{3}{|c|}{ High school } \\
\hline & & & Male & Female & Junior & Senior & Vocational \\
\hline \multicolumn{8}{|l|}{ SHS home } \\
\hline No (Ref.) & $3.1(2.7-3.6)$ & 1 & 1 & 1 & 1 & 1 & 1 \\
\hline Yes & $5.1(4.5-5.8)$ & $1.46(1.14-1.87)$ & $1.73(1.25-2.40)$ & $1.13(0.76-1.67)$ & $2.00(1.32-3.03)$ & $0.95(0.61-1.48)$ & $1.28(0.90-1.83)$ \\
\hline \multicolumn{8}{|l|}{ SHS public } \\
\hline No (Ref.) & $3.5(3.0-4.2)$ & 1 & 1 & 1 & 1 & 1 & 1 \\
\hline Yes & $4.1(3.7-4.6)$ & $0.97(0.76-1.23)$ & $0.93(0.68-1.28)$ & $0.99(0.69-1.43)$ & $0.95(0.61-1.48)$ & $0.94(0.61-1.44)$ & $1.51(1.07-2.15)$ \\
\hline \multicolumn{8}{|l|}{ SHS level } \\
\hline Low (Ref.) & $3.0(2.5-3.6)$ & 1 & 1 & 1 & 1 & 1 & 1 \\
\hline Medium & $3.7(3.1-4.3)$ & $1.18(0.92-1.52)$ & $1.31(0.94-1.83)$ & $1.02(0.70-1.50)$ & $1.42(0.95-2.13)$ & $0.86(0.55-1.36)$ & $1.31(0.89-1.93)$ \\
\hline High & $5.5(4.7-6.3)$ & $1.51(1.16-1.97)$ & $1.66(1.18-2.33)$ & $1.27(0.82-1.97)$ & $1.51(0.97-2.34)$ & $1.28(0.79-2.07)$ & $1.83(1.22-2.75)$ \\
\hline$p$ for trend & & 0.001 & 0.002 & 0.190 & 0.026 & 0.243 & 0.009 \\
\hline SHS (cont.) & & $1.04(1.01-1.08)$ & $1.06(1.02-1.10)$ & $1.02(0.97-1.07)$ & $1.04(1.00-1.09)$ & $1.04(0.98-1.11)$ & $1.05(1.00-1.09)$ \\
\hline \multicolumn{8}{|c|}{ SHS home and public } \\
\hline No (Ref.) & $3.3(2.7-4.0)$ & 1 & 1 & 1 & 1 & 1 & 1 \\
\hline Home only & $4.7(3.2-6.9)$ & $1.43(0.86-2.36)$ & $1.78(0.96-3.31)$ & $0.93(0.38-2.31)$ & $2.90(1.50-5.64)$ & $0.55(0.16-1.82)$ & $0.65(0.27-1.53)$ \\
\hline Public only & $3.0(2.5-3.6)$ & $0.97(0.73-1.29)$ & $0.96(0.65-1.41)$ & $0.94(0.62-1.43)$ & $0.97(0.61-1.54)$ & $0.83(0.51-1.36)$ & $1.26(0.81-1.97)$ \\
\hline Both & $5.2(4.5-5.9)$ & $1.43(1.07-1.90)$ & $1.64(1.13-2.38)$ & $1.12(0.71-1.78)$ & $1.69(1.03-2.77)$ & $0.87(0.53-1.45)$ & $1.90(1.23-2.95)$ \\
\hline
\end{tabular}

OR: odds ratio. a Model adjusted for age, sex, school type, district, boarding, local, GPA, pocket money, friends' smoking and parents' smoking. 
Table 5. ORs for e-cigarette use by secondhand smoke exposure stratified by sex and school $(\mathrm{N}=12278)^{b}$

\begin{tabular}{|c|c|c|c|c|c|c|c|}
\hline & \multirow{2}{*}{$\begin{array}{c}\text { E-cigarette use } \\
\%(95 \% \mathrm{CI})\end{array}$} & \multirow[t]{2}{*}{ Total } & \multicolumn{2}{|c|}{ Sex } & \multicolumn{3}{|c|}{ High school } \\
\hline & & & Male & Female & Junior & Senior & Vocational \\
\hline \multicolumn{8}{|l|}{ SHS home } \\
\hline No (Ref.) & $3.2(2.8-3.6)$ & 1 & 1 & 1 & 1 & 1 & 1 \\
\hline Yes & $7.5(6.8-8.2)$ & $1.53(1.24-1.89)$ & $1.42(1.11-1.81)$ & $1.82(1.18-2.79)$ & $1.27(0.88-1.84)$ & $1.67(1.07-2.6)$ & $1.81(1.36-2.42)$ \\
\hline \multicolumn{8}{|l|}{ SHS public } \\
\hline No (Ref.) & $2.9(2.4-3.5)$ & 1 & 1 & 1 & 1 & 1 & 1 \\
\hline \multicolumn{8}{|l|}{ SHS level } \\
\hline Low (Ref.) & $2.6(2.2-3.1)$ & 1 & 1 & 1 & 1 & 1 & 1 \\
\hline Medium & $3.8(3.3-4.4)$ & $1.28(1.00-1.64)$ & $1.20(0.90-1.61)$ & $1.54(0.95-2.47)$ & $1.43(0.93-2.21)$ & $1.56(0.84-2.90)$ & $1.08(0.79-1.47)$ \\
\hline High & $9.4(8.5-10.4)$ & $2.25(1.77-2.87)$ & $1.99(1.50-2.64)$ & $3.05(1.90-4.87)$ & $2.33(1.52-3.56)$ & $4.32(2.41-7.74)$ & $1.45(1.07-1.96)$ \\
\hline$p$ for trend & & $<0.001$ & $<0.001$ & $<0.001$ & $<0.001$ & $<0.001$ & 0.019 \\
\hline SHS (cont.) & & $1.10(1.07-1.12)$ & $1.08(1.05-1.12)$ & $1.12(1.07-1.18)$ & $1.09(1.05-1.14)$ & $1.18(1.12-1.25)$ & $1.03(1.00-1.07)$ \\
\hline \multicolumn{8}{|c|}{ SHS home and public } \\
\hline Home only & $2.9(1.9-4.2)$ & $0.94(0.58-1.52)$ & $0.80(0.45-1.44)$ & $1.34(0.56-3.20)$ & $0.43(0.13-1.47)$ & $0.47(0.06-3.89)$ & $1.65(0.94-2.88)$ \\
\hline Public only & $3.4(2.9-4.0)$ & $1.13(0.85-1.49)$ & $1.09(0.78-1.52)$ & $1.23(0.72-2.10)$ & $1.09(0.70-1.71)$ & $1.83(0.92-3.64)$ & $0.89(0.61-1.30)$ \\
\hline Both & $8.1(7.4-8.9)$ & $1.89(1.44-2.47)$ & $1.72(1.25-2.36)$ & $2.36(1.37-4.06)$ & $1.58(0.99-2.54)$ & $3.36(1.70-6.65)$ & $1.66(1.19-2.32)$ \\
\hline
\end{tabular}

OR: odds ratio. b: Model adjusted for age, sex, school type, district, boarding, local, GPA, pocket money, friends' smoking, parents' smoking and smoking status. 


\section{DISCUSSION}

Our study showed a still high prevalence of SHS exposure among adolescents in Shanghai, China, and the positive association of SHS exposure, especially dual exposure at home and public places, with tobacco use behaviors. We also found the gender and school type disparities in SHS exposure and smoking behaviors and the extent to which these disparities are pronounced over exposure level/source. Our findings might supplement the evidence on the impacts of SHS exposure in adolescents, providing reference for the formulation of targeted prevention and control strategies.

The exposure prevalence of SHS at home and indoor public places among adolescents in Shanghai (41.5\% and 59.3\%) are apparently lower than those obtained from China's 2019 national survey (63.2\% and $72.0 \%)^{10}$. Young populations in our study reported the highest exposure rate at outdoor areas $(63.5 \%)$, but this rate is also lower than the national level $(67.3 \%)$. These relatively low exposure rates could be explained by local policies. The Regulations of Shanghai Municipality on smoking control in public places came into effect 1 March 2017 and banned smoking in all indoor areas. Therefore, parents might have better awareness and knowledge in avoiding smoking in families, especially in the presence of their children ${ }^{26}$. However, the indoor clean air laws lacked effective supervision and smoking control at outdoor public places, which remained the main sources of exposure to SHS among adolescents in Shanghai. The SHS exposure rate of adolescents in China is significantly higher than those in developed countries (e.g. home exposure rate $21.7 \%$ in the US) ${ }^{13}$.

Exposure to SHS is an independent risk factor of being susceptible to smoking in adolescents. This study indicates that non-smokers who were exposed to SHS at home and in public places had a higher prevalence of susceptibility to smoking than those who were not and the rate increased by level of SHS exposure. These findings are consistent with prior studies ${ }^{27}$.

The separate and combined prevalence of SHS exposure from different sources were studied. It is worth noting that SHS exposure rate from only one source, either home or public place, is relatively low but the exposure rate from multiple sources is surprisingly high. Our study also shows that
SHS exposure at one only place had little impact, but exposure at two places significantly increased smoking-related behaviors. This indicates that adolescents are more likely to be exposed to SHS both at home and at public places. Exposed to SHS in multiple places may greatly increase risk of tobacco use among youth.

This study shows SHS exposure source disparities in their impacts on smoking behaviors. Adolescents exposed to household SHS have a higher current smoking risk than those exposed at public places. In terms of smoking susceptibility, exposure to SHS at home also has a greater impact on non-smokers compared with public places. This suggests that SHS exposure at home is more likely to have both immediate and long-term effects on youth, initiating non-smokers into smoking onset, in line with the previous research ${ }^{28}$. Prior studies have shown that adolescents with parental smoking are more likely to be exposed to home $\mathrm{SHS}^{29}$. Parental smoking was associated with a higher risk of initiating adolescent offspring into smoking ${ }^{30}$ and SHS exposure may explain the relationship between parental smoking and the beginning of smoking ${ }^{27}$. Considering these conditions, special smoke-free home interventions and strengthening parents' family anti-smoking awareness may prove effective in restricting adolescents from becoming active smokers from SHS exposure ${ }^{31-33}$.

Our results on impacts of exposure to SHS from different sources on adolescent smoking behaviors show differences in gender and school. Boys exposed to SHS at home or at public places were more significantly affected in terms of current smoking use than girls who conversely had a higher prevalence of SHS exposure in public places. This conclusion is similar to that of the existing studies ${ }^{22,28}$. It might be due to the social perception that smoking among males is more acceptable than among females. Boys emulating the behaviors of adult males with higher smoking rate, are more likely to initiate smoking ${ }^{34}$. Although SHS exposure was not significantly related to smoking susceptibility in girls, females who were exposed to domestic and high-level SHS had higher odds of smoking currently. The focus of tobacco control in females had changed from SHS in the 1990 s to both smoking and SHS in $2017^{35}$. Studies have suggested that there has been a steady increase in smoking prevalence among young females in China, 
from $0.29 \%$ in 1984 to $18.1 \%$ in $2018^{35,36}$. Thus, further research targeting factors that influence girl's smoking behaviors and practical strategies focusing on preventing female adolescents from initiating smoking, are urgently needed.

The risk of current smoking and smoking susceptibility among junior high school students was more significantly affected by SHS exposure at home. According to the theory of human development, family members have a stronger influence on adolescents aged 13-15 years than on those aged 16-17 years ${ }^{37}$. Junior high school students who are younger are likely to imitate the smoking behaviors of intimate people, especially parents. SHS exposure in public places has a greater impact on future susceptibility to smoking for vocational school students, which might be due to the fact that they have more contact with society than other school students, and are thus more susceptible to deleterious environmental influences.

E-cigarette use among youth has gained popularity worldwide ${ }^{38}$ and the prevalence of e-cigarette use among middle school students in China is raising $(1.2 \% \text { in } 2014 \text { to } 2.7 \% \text { in } 2019)^{39}$. Identifying youth who are susceptible to e-cigarette use might help in prevention efforts. Current studies reveal that e-cigarette secondhand aerosol (SHA) exposure was associated with susceptibility to e-cigarette $u^{40}{ }^{40}$. Our study adds to this that conventional combustible tobacco smoke exposure is also associated with e-cigarette use among almost all groups of adolescents. Females and senior high school students have greater odds of using e-cigarettes.

Considering the strong effects observed of SHS exposure on adolescents' smoking behaviors, it is necessary to better control the exposure of susceptible adolescents to SHS. Especially, strategic interventions for non-smokers exposed to SHS who are at high risk of smoking susceptibility and prohibiting the transition from susceptibility to smoking behavior, may be an effective smoking prevention measure. As stated by the WHO, only eliminating smoking in indoor spaces fully can protect non-smokers from exposure to SHS. Given the high rate of SHS exposure at outdoor areas, there is concern that the smoking bans should be considered more comprehensively, covering not only indoor workplaces and public places. Apart from general tobacco control legislations, policies should emphasize the protection of adolescents' health, and impose stringent tobacco control regulations in schools and other places where the minors gather.

\section{Strengths and limitations}

The cross-sectional study design is a limitation, as a causal link between study variables and current smoking status and susceptibility to smoking cannot be established. How factors during adolescence assist in predicting smoking in later adolescence needs clarification using a longitudinal design. Future studies are warranted to compile longitudinal surveillance data to examine smoking susceptibility and initiation. Our study might have been biased, as some possible potential confounding factors were not controlled for. However, the representativeness, large sample size and high response rate among students, enables generalization of the results to the schoolgoing adolescent population in China.

\section{CONCLUSIONS}

Exposure to SHS has significant impact on tobacco use in the younger population, irrespective of gender and type of school. Considering the health and social consequences associated with SHS exposure, tailored public health policies and measures such as health promotion activities and strong anti-smoking regulations are needed to increase awareness of the adverse health effects of smoking, especially among adolescent populations. Society, schools and families should jointly work to create a smoke-free environment, and develop and implement policies for preventing cigarette use among adolescents.

\section{REFERENCES}

1. Thacher JD, Schultz ES, Hallberg J, et al. Tobacco smoke exposure in early life and adolescence in relation to lung function. Eur Respir J. 2018;51(6):1702111. doi:10.1183/13993003.02111-2017

2. Milanzi EB, Koppelman GH, Smit HA, Wijga AH, Vonk JM, Brunekreef B, Gehring U. Timing of secondhand smoke, pet, dampness or mould exposure and lung function in adolescence. Thorax. 2019;75(2):153-163. doi:10.1136/thoraxjnl-2019-213149

3. Raghuveer G, White DA, Hayman LL, et al. Cardiovascular Consequences of Childhood Secondhand Tobacco Smoke Exposure: Prevailing Evidence, Burden, and Racial and Socioeconomic Disparities: A Scientific Statement From the American Heart Association. Circulation. 2016;134(16):e336-e359. doi:10.1161/cir.0000000000000443

4. Pierce JP, Choi WS, Gilpin EA, Farkas AJ, Merritt RK. 
Validation of susceptibility as a predictor of which adolescents take up smoking in the United States. Health Psychol. 1996;15(5):355-361. doi:10.1037//0278-6133.15.5.355

5. El-Toukhy S, Sabado M, Choi K. Trends in Susceptibility to Smoking by Race and Ethnicity. Pediatrics. 2016;138(5):e20161254. doi:10.1542/peds.2016-1254

6. Wills TA, Knight R, Williams RJ, Pagano I, Sargent JD. Risk factors for exclusive e-cigarette use and dual e-cigarette use and tobacco use in adolescents. Pediatrics. 2015;135(1):e43-e51. doi:10.1542/peds.2014-0760

7. Li VC, Tang S. China's New Road for Tobacco Control: Tobacco Crop Substitution. Am J Public Health. 2018;108(10):1316-1317. doi:10.2105/ajph.2018.304620

8. Yang G, Wang Y, Wu Y, Yang J, Wan X. The road to effective tobacco control in China. Lancet. 2015;385(9972):10191028. doi:10.1016/S0140-6736(15)60174-X

9. Barrington-Trimis JL, Braymiller JL, Unger JB, et al. Trends in the Age of Cigarette Smoking Initiation Among Young Adults in the US From 2002 to 2018. JAMA Netw Open. 2020;3(10):e2019022. doi:10.1001/jamanetworkopen.2020.19022

10. Chinese Center for Disease Control and Prevention. [2019 China's National Youth Tobacco Survey Result]. Accessed May 31, 2020. http://www.chinacdc.cn/ yw_9324/202005/t20200531_216942.html

11. Epperson AE, Wallander JL, Song AV, et al. Gender and racial/ethnic differences in adolescent intentions and willingness to smoke cigarettes: Evaluation of a structural equation model. J Health Psychol. 2019;26(4):605-619. doi:10.1177/1359105319829536

12. Agaku IT, Singh T, Rolle I, Olalekan AY, King BA. Prevalence and Determinants of Secondhand Smoke Exposure Among Middle and High School Students. Pediatrics. 2016;137(2):e20151985. doi:10.1542/peds.2015-1985

13. Bayly JE, Bernat D, Porter L, O'Dare K, Choi K. Prevalence and characteristics of secondhand smoke and secondhand vapour exposure among youth. Tob Control. 2019;28(3):305310. doi:10.1136/tobaccocontrol-2018-054265

14. Okoli CT, Kelly T, Hahn EJ. Secondhand smoke and nicotine exposure: a brief review. Addict Behav. 2007;32(10):19771988. doi:10.1016/j.addbeh.2006.12.024

15. Lessov-Schlaggar CN, Wahlgren DR, Liles S, et al. Sensitivity to secondhand smoke exposure predicts future smoking susceptibility. Pediatrics. 2011;128(2):254-262. doi:10.1542/peds.2010-3156

16. Bélanger M, O'Loughlin J, Okoli CT, et al. Nicotine dependence symptoms among young never-smokers exposed to secondhand tobacco smoke. Addict Behav. 2008;33(12):1557-1563. doi:10.1016/j.addbeh.2008.07.011

17. Okoli CT, Kodet J. A systematic review of secondhand tobacco smoke exposure and smoking behaviors: Smoking status, susceptibility, initiation, dependence, and cessation. Addict Behav. 2015;47:22-32. doi:10.1016/j.addbeh.2015.03.018

18. Solomon RL, Corbit JD. An opponent-process theory of motivation. II. Cigarette addiction. J Abnorm Psychol. 1973;81(2):158-171. doi:10.1037/h0034534

19. Lewinsohn PM, Rohde P, Brown RA. Level of current and past adolescent cigarette smoking as predictors of future substance use disorders in young adulthood. Addiction. 1999;94(6):913-921. doi:10.1046/j.1360-0443.1999.94691313.x

20. Leung LT, Ho SY, Wang MP, Lam TH. Secondhand Smoke From Multiple Sources, Thirdhand Smoke and Respiratory Symptoms in Hong Kong Adolescents. Nicotine Tob Res. 2018;20(2):192-198. doi:10.1093/ntr/ntw302

21. Makadia LD, Roper PJ, Andrews JO, Tingen MS. Tobacco Use and Smoke Exposure in Children: New Trends, Harm, and Strategies to Improve Health Outcomes. Curr Allergy Asthma Rep. 2017;17(8):55. doi:10.1007/s11882-017-0723-0

22. Veeranki SP, Mamudu HM, Zheng S, et al. Secondhand smoke exposure among never-smoking youth in 168 countries. J Adolesc Health. Feb 2015;56(2):167-73. doi:10.1016/j.jadohealth.2014.09.014

23. Nuño VL, Zhang Q, Harris RB, Wilkinson-Lee AM, Wilhelm MS. Smoking susceptibility among students followed from grade six to eight. Addict Behav. 2011;36(12):1261-1266. doi:10.1016/j.addbeh.2011.07.041

24. Zhang X, Pu J. E-cigarette use among US adolescents: secondhand smoke at home matters. Int J Public Health. 2016;61(2):209-213. doi:10.1007/s00038-015-0784-6

25. Zhu J, Li J, He Y, Li N, Xu G, Yu J. The influence and interaction of exposure to pro-smoking and antismoking messaging on youth smoking behaviour and susceptibility. Tob Induc Dis. 2019;17(November). doi:10.18332/tid/114066

26. Cheng KW, Okechukwu CA, McMillen R, Glantz SA. Association between clean indoor air laws and voluntary smokefree rules in homes and cars. Tob Control. 2015;24(2):168-174. doi:10.1136/tobaccocontrol-2013-051121

27. McGrath JJ, Racicot S, Okoli CTC, Hammond SK, O'Loughlin J. Airborne Nicotine, Secondhand Smoke, and Precursors to Adolescent Smoking. Pediatrics. 2018;141(Suppl 1):S63-S74. doi:10.1542/peds.2017-1026J

28. Lee KA, Palipudi KM, English LM, Ramanandraibe N, Asma S; GYTS collaborative group. Secondhand smoke exposure and susceptibility to initiating cigarette smoking among never-smoking students in selected African countries: Findings from the Global Youth Tobacco Survey. Prev Med. 2016;91S:S2-S8. doi:10.1016/j.ypmed.2016.04.017

29. Hwang JH, Park SW. Sex and Age Differences in Exposure to Secondhand Smoke at Home among Korean Adolescents: A Nationally Representative Survey. Int J Environ Res Public Health. 2016;13(2):241. doi:10.3390/ijerph13020241

30. Gilman SE, Rende R, Boergers J, et al. Parental smoking and adolescent smoking initiation: an intergenerational perspective on tobacco control. Pediatrics. 
2009;123(2):e274-e281. doi:10.1542/peds.2008-2251

31. Turner S, Mackay D, Dick S, Semple S, Pell JP. Associations between a smoke-free homes intervention and childhood admissions to hospital in Scotland: an interrupted time-series analysis of whole-population data. Lancet Public Health. 2020;5(9):e493-e500. doi:10.1016/S2468-2667(20)30178-X

32. Rosen LJ, Myers V, Winickoff JP, Kott J. Effectiveness of Interventions to Reduce Tobacco Smoke Pollution in Homes: A Systematic Review and Meta-Analysis. Int J Environ Res Public Health. 2015;12(12):16043-16059. doi:10.3390/ijerph121215038

33. Snyder K, Vick JH, King BA. Smoke-free multiunit housing: a review of the scientific literature. Tob Control. 2016;25(1):920. doi:10.1136/tobaccocontrol-2014-051849

34. Lim KH, Ghazali SM, Lim HL, et al. Smoking susceptibility among non-smoking school-going adolescents in Malaysia: findings from a national school-based survey. BMJ Open. 2019;9(10):e031164. doi:10.1136/bmjopen-2019-031164

35. Wen H, Xie C, Wang F, Wu Y, Yu C. Trends in Disease Burden Attributable to Tobacco in China, 1990-2017: Findings From the Global Burden of Disease Study 2017. Front Public Health. 2020;8:237. doi:10.3389/fpubh.2020.00237

36. Wang M, Luo X, Xu S, et al. Trends in smoking prevalence and implication for chronic diseases in China: serial national cross-sectional surveys from 2003 to 2013. Lancet Respir Med. 2019;7(1):35-45. doi:10.1016/s2213-2600(18)30432-6

37. Masiero M, Lucchiari C, Pravettoni G. Personal fable: optimistic bias in cigarette smokers. Int J High Risk Behav Addict. 2015;4(1):e20939. doi:10.5812/ijhrba.20939

38. Singh T, Arrazola RA, Corey CG, et al. Tobacco Use Among Middle and High School Students--United States, 2011-2015. MMWR Morb Mortal Wkly Rep. 2016;65(14):361-367. doi:10.15585/mmwr.mm6514a1

39. Xiao L, Parascandola M, Wang C, Jiang Y. Perception and Current Use of E-cigarettes Among Youth in China. Nicotine Tob Res. 2019;21(10):1401-1407. doi:10.1093/ntr/nty145

40. Agaku IT, Perks SN, Odani S, Glover-Kudon R. Associations between public e-cigarette use and tobacco-related social norms among youth. Tob Control. 2020;29(3):332-340. doi:10.1136/tobaccocontrol-2018-054728

\section{CONFLICTS OF INTEREST}

The authors have each completed and submitted an ICMJE form for disclosure of potential conflicts of interest. The authors declare that they have no competing interests, financial or otherwise, related to the current work. All the authors report that they received the Grant No. 20BSH133 from the National Social Science Foundation of China and the Grant No. GWV-10.1-XK15 from the Shanghai Key Discipline Construction Project in Public Health.

\section{FUNDING}

This work was supported by the grants from National Social Science Foundation of China (Grant No. 20BSH133) and Shanghai Key Discipline Construction Project in Public Health (Grant No. GWV-10.1-XK15).

\section{ETHICAL APPROVAL AND INFORMED CONSENT}

This study was approved by the Ethics Committee of the Shanghai Jiao Tong University (SJUPN-201703; approved on December 5, 2017). Completion of the online questionnaire was taken as informed consent.

\section{DATA AVAILABILITY}

The data supporting this research are available from the authors on reasonable request.

\section{AUTHORS' CONTRIBUTIONS}

JZ designed the study and revised this manuscript. XY and ZY carried out data analysis, result interpretation, and manuscript preparation. $\mathrm{GX}$ and YT collected data. All authors have read and approved the final manuscript.

PROVENANCE AND PEER REVIEW

Not commissioned; externally peer reviewed. 